\title{
Cocultures of Peronospora tabacina and Nicotiana Species to Study Host-Pathogen Interactions
}

\author{
E. P. Heist, W. C. Nesmith, and C. L. Schardl
}

Department of Plant Pathology, University of Kentucky, Lexington 40546.

Accepted for publication 29 August 2001.

\begin{abstract}
Heist, E. P., Nesmith, W. C., and Schardl, C. L. 2001. Cocultures of Peronospora tabacina and Nicotiana species to study host-pathogen interactions. Phytopathology 91:1224-1230.

Long-term cocultures of the tobacco blue mold pathogen, Peronospora tabacina, with Nicotiana tabacum and $N$. repanda callus were derived from infected host plant tissue. In this apparently contaminant-free system, sporulation occurred under similar conditions as in intact plants. Sporangia were collected from cocultures and used to complete Koch's postulates. The cocultures were grown under two light regimes. One

phores and sporangia. Production of normal sporangiophores and sporangia was achieved by transferring light-grown cocultures to overnight darkness and resulted in necrosis of the callus. Cocultures of Peronospora tabacina with either host species, grown in total darkness, frequently sporulated with minimal necrosis over the course of 1 year. Thus, cocultures should prove useful as a source of Peronospora tabacina over extended periods of time at low risk of pathogen release, for studying the physiology of Peronospora tabacina-Nicotiana interactions, maintaining Peronospora tabacina lines for genetic studies, and providing a reliable source of axenic inoculum for research.
\end{abstract} consisted of $23 \mathrm{~h}$ of light followed by $1 \mathrm{~h}$ of darkness and the second comprised total darkness. Sporulation occurred frequently in the $23 \mathrm{~h}$ light-grown cocultures but resulted in production of abnormal sporangio-
Additional keywords: downy mildew, gnotobiotic, oomycete, tissue culture.
Peronospora tabacina D. B. Adam, the pathogen responsible for downy mildew (blue mold) of tobacco, is an obligate biotroph that infects many species in the genus Nicotiana. Species of Nicotiana vary from immune to highly susceptible to blue mold (3). Included among the hosts regarded as highly susceptible is $N$. tabacum (tobacco), which is grown commercially in the United States and worldwide. Another host to the blue mold pathogen, characterized as tolerant to the disease, is $N$. repanda. This host is a wild relative of tobacco found in the southwestern United States and Mexico, and has been implicated as one of the primary overwintering hosts of Peronospora tabacina (18).

Peronospora tabacina is an oomycete. Examples of other closely related phytopathogenic oomycetes include Pythium and Phytophthora spp. $(6,7)$, which cause such diseases as damping off and root rot, respectively. Unlike many well-studied plant pathogens that may be isolated and cultured on nutrient media, Peronospora tabacina cannot complete its life cycle apart from living host tissue. Traditionally, the study of blue mold involves harvesting sporangia from an infected plant and using them to inoculate and infect susceptible hosts. The inherent difficulties in studying an obligate biotroph are compounded by the need for careful containment, especially when working in tobacco production areas, because the pathogen produces abundant airborne sporangia that could potentially serve as primary inoculum and cause local epiphytotics. Conditions for sporulation of Peronospora tabacina on infected plants include high relative humidity (>95\%), temperatures in the range of 18 to $23^{\circ} \mathrm{C}$, and an overnight period of darkness (19).

The primary objective of this study was to determine whether Peronospora tabacina can reliably establish long-term infections of callus from wild and cultivated tobacco, specifically $N$. repanda

Corresponding author: E. P. Heist; E-mail address: epheis1@uky.edu

Publication no. P-2001-1016-01R

(c) 2001 The American Phytopathological Society and $N$. tabacum cv. KY14. Because the oomycetes that cause downy mildew diseases are obligate biotrophs, one option for culturing them for research has been on their respective hosts in tissue culture (4). This can be accomplished in two ways: one involves the inoculation of axenic callus of the host with sporangia from the pathogen, and the other involves initiating the cocultures from host tissues already infected by the pathogen (4). Morel (9) established the first dual cultures of a downy mildew pathogen (Plasmopara viticola) and its host (Vitis vinifera) by inoculating callus tissue of grapevine with aseptic zoospores of Plasmopara viticola. Izard et al. (5) obtained dual tissue cultures of Peronospora tabacina and various Nicotiana spp. (including a susceptible burley tobacco hybrid) in a similar manner by inoculating axenic callus tissue with sporangia from the pathogen. These infections of tobacco callus could only be detected by microscopy and were characterized by the localization of mycelium, multiplication of haustoria, and scant sporulation or emergence of sterile aerial hyphae. These studies were expanded on by Trigiano et al. (14) who compared the infection process of tobacco callus with that of intact plants. The method employed, previously described by Amerson and Mott (1), involved inoculating axenic tobacco callus with Peronospora tabacina sporangia, and resulted in only sparse mycelia with no observable sporulation. Within 20 days, only a few hyphal cells were observable, indicating rapid decline of the pathogen. Thus, sustained propagation of Peronospora tabacina outside of intact plants has not been reported previously. In this paper, we describe how to establish and maintain, for extended periods, cocultures of two Nicotiana spp. and Peronospora tabacina. During the course of this investigation, other observations were made with regard to host-pathogen interactions and the general biology of the system, which will be discussed.

\section{MATERIALS AND METHODS}

Host material. Burley tobacco plants (N. tabacum cv. KY14) and the wild tobacco species, $N$. repanda (seed provided by the 
Oxford Tobacco Research Station at North Carolina State University), were grown from seed in 6-inch pots containing Pro Mix Bx soil mix (Premier Horticulture Inc., Red Hill, PA). Plants were maintained in growth chambers at $23^{\circ} \mathrm{C}$ and watered, as needed, with a $4 \mathrm{ml} / \mathrm{liter}$ of Peter's brand 20-10-20 fertilizer (Horticultural Products Company, Marysville, $\mathrm{OH})$. Axenic plants for use in gnotobiotic associations (featuring axenic whole-plants inoculated with Peronospora tabacina) were derived from surface-sterilized $N$. tabacum (cv. KY14) and N. repanda seed. Seeds were surfacesterilized by placing for $3 \mathrm{~min}$ in $70 \%$ ethanol, $30 \mathrm{~min}$ in a $1.5 \%$ solution of sodium hypochlorite, followed by two sterile water rinses. For germination, surface-sterilized seeds were plated on MS-, an MS (Murashige and Skoog; 10) basal salts medium with no growth hormones. For subsequent growth or inoculation, seedlings were transferred to fresh MS- medium, two plants to a plate.

Production of inoculum and plant inoculations. Isolate KY79 of Peronospora tabacina (12) was used for all inoculations in this study. This isolate was collected from a tobacco field near Georgetown, Kentucky, in 1979, and was experimentally determined to be sensitive to the fungicide, metalaxyl. The pathogen was maintained on 8- to 14 -week-old N. tabacum cv. KY14 plants grown in growth chambers at $23^{\circ} \mathrm{C}$. Infected plants were maintained by infiltrating pathogen spores into leaves; the pathogen could be induced to sporulate 7 to 9 days postinoculation by placing infected leaves in a moistened, black plastic bag overnight. Bags were moistened by spraying a fine water mist inside prior to insertion of infected leaves. Sporangia were harvested the following day by lightly scraping the surface of sporulating lesions with a sterile wooden applicator stick (Fisher Scientific, Pittsburgh, PA) and swirling in a $1.5-\mathrm{ml}$ microcentrifuge tube containing $1 \mathrm{ml}$ of sterile distilled water. New 8- to 14-week-old $N$. tabacum plants were inoculated by placing several $10-\mu$ droplets of a $5 \times 10^{3}$ spores per $\mathrm{ml}$ (determined with a hemacytometer) suspension onto leaf panels. The plants were then placed in moistened, black plastic bags overnight to support infection. All steps were carried out with due precaution for containing the pathogen and minimizing potential for contamination.

Preparation of tissue culture media. Two types of media were used to grow cocultures and axenic host plants. These included MS- and MS+ (MS+ with plant growth hormones). MS+ (10) contained $25 \mathrm{mg}$ of 6-benzylaminopurine (BAP), $1 \mathrm{mg}$ of indole 3 -acetic acid (IAA), $30 \mathrm{~g}$ of sucrose, and $9 \mathrm{~g}$ of phytagar (tissue culture grade, Gibco BRL, Grand Island, NY) per liter. The $\mathrm{pH}$ was adjusted between 5.7 to 5.9 with $1 \mathrm{M} \mathrm{NaOH}$ and sterilized by autoclaving. Antibiotics, added to the sterile medium cooled to around $48^{\circ} \mathrm{C}$, included $2.5 \mathrm{mg}$ of carbenicillin per liter and $5 \mathrm{mg}$ of cefotaxime per liter (both from 1,000X stocks filter sterilized with a $0.22 \mu \mathrm{m}$ Millipore filter; Millipore S.A., Molsheim, France). MS- medium, used for growing axenic plants and inducing root formation, was prepared as described for MS+ medium, except no BAP or IAA was added and only $20 \mathrm{~g}$ of sucrose per liter was used.

Development of cocultures with surface-sterilized infected leaf tissue. Disks, 3 to $4 \mathrm{~cm}$ in diameter, were excised from chlorotic lesions (5 days postinoculation) of Peronospora tabacinainfected $N$. tabacum plants, and surface-sterilized by submerging for $30 \mathrm{~s}$ in $70 \%$ ethanol, followed by transfer to $0.5 \%$ sodium hypochlorite for $10 \mathrm{~min}$, and two rinses in sterile distilled water. The surface-sterilized infected tissue was then further subdivided into square pieces $(0.5 \mathrm{~cm}$ to a side) and placed (five pieces to a plate $)$ on five separate petri plates $(25 \times 100 \mathrm{~mm})$ containing MS+ medium. Four of the plates were sealed with Parafilm and were incubated at $23^{\circ} \mathrm{C}$ in a growth chamber with a $23 \mathrm{~h}$ photoperiod. Light intensity was measured at $66 \mu \mathrm{E} / \mathrm{m}^{2} / \mathrm{s}$. The fifth plate was sealed and incubated overnight in the dark at room temperature and observed the following day for sporulation of the pathogen to confirm that the pathogen was present in the tissue and had survived the surface-sterilization procedure. The remaining four plates were maintained in $23 \mathrm{~h}$ light and subcultured onto fresh MS+ medium every 2 weeks.

Development of Peronospora tabacina infections on axenic Nicotiana spp. Using a sterile hypodermic syringe with a 25gauge needle, a spore suspension obtained from cocultures derived from surface-sterilized infected leaf tissue was applied, dropwise $(\approx 10$ - to 20 - $\mu$ l aliquots), onto the foliage of five 6- to 8 -week-old axenic $N$. tabacum cv. KY14 and N. repanda plants. Axenic plants were derived from surface-sterilized seed as described previously. Inoculated plants were maintained in growth chambers (Model CU-36L5; Percival Scientific Inc., Boone, IA) at $23^{\circ} \mathrm{C}$ with a $23 \mathrm{~h}$ photoperiod. Plants were grown in petri plates sealed with Parafilm to maintain axenic conditions. Once successful infection of axenic host plants was confirmed, cocultures derived from surface-sterilized infected leaf tissue were autoclaved and discarded. Inoculum was obtained from the whole-plant system by inducing sporulation in overnight darkness and used to inoculate new axenic host plants. This procedure was repeated to propagate Peronospora tabacina on axenic host plants.

Initiation of new cocultures from gnotobiotic infections and maintaining cocultures. Fifty pieces of infected (chlorotic) leaf tissue (squares, $0.5 \mathrm{~cm}$ to a side) were obtained by excision from each combination of host and pathogen from gnotobiotic associations 10 days postinoculation and plated directly onto MS+ medium. The 50 pieces of infected leaf tissue from each host species were divided into two groups of 25 (each group having five plates with five pieces per plate). One group was placed in $24 \mathrm{~h}$ dark and the other in $23 \mathrm{~h}$ light at $23^{\circ} \mathrm{C}$. Plates that were placed in $24 \mathrm{~h}$ dark were exposed to the light only during biweekly subculturing and periodic observation. Of the five plates of infected leaf tissue per treatment, four plates (total $=20$ pieces of infected callus) were transferred to fresh MS+ medium every 2 weeks. The remaining plate (with five pieces of infected callus) from each treatment was left on the original medium for observation without subsequent transfer. Likewise, controls with healthy tissue from both axenic $N$. repanda and $N$. tabacum were plated on MS+ medium and treated as described previously. Except where specified, results reflect cocultures that were subcultured to fresh medium every 2 weeks. The time course of this study was 1 year, after which all tissue was autoclaved and discarded.

Tests for contamination in cocultures. To check for contamination of sporangia harvested from cocultures, sporulation was induced on some of the light-grown cocultures, once a month, beginning the first month after plating infected leaf tissue. Likewise, representative cocultures were collected from dark-grown callus of each host species on which sporulation was observed. Sporangial suspensions were then collected as previously described and added to each of 10 culture tubes, half containing $5 \mathrm{ml}$ of potato dextrose broth (PDB; Becton Dickinson and Company, Sparks, MD) and the other half containing $5 \mathrm{ml}$ of nutrient broth (NB; Becton Dickinson and Company, Cockeysville, MD). The tubes were incubated at $25^{\circ} \mathrm{C}$ for 2 months and checked weekly for contaminating bacteria and fungi by comparing with uninoculated media (negative controls) and by examining small aliquots (20 to $50 \mu \mathrm{l}$ ) microscopically. In addition, $2 \mathrm{~g}$ of coculture material that exhibited Peronospora tabacina sporulation was obtained from each host species and from each light regime and placed into a sterile plastic bag with $20 \mathrm{ml}$ of sterile water and pulverized manually. This material was then spread (in $0.5-\mathrm{ml}$ aliquots) onto each of five plates of potato dextrose agar (PDA) and five plates of nutrient agar (NA; NB with 2\% Bacto agar; Becton Dickinson and Company, Sparks, MD) and put (in 0.5-ml aliquots) into each of five tubes containing $5 \mathrm{ml}$ of PDB and into five tubes containing $5 \mathrm{ml}$ of NB. All plates and tubes were labeled and incubated for 2 months and monitored regularly for microbial contamination (both visually and microscopically). Positive controls consisted of Peronospora tabacina-infected cv. KY14 leaf material from greenhouse-grown plants placed into 
each of the four media (PDB, NB, PDA, and NA), and the negative controls consisted of representatives of each medium both with uninfected axenic host material and without any introduced tissue.

Koch's postulates for proof of pathogenicity. To confirm that sporangia produced from cocultures were indeed those of Peronospora tabacina, modified Koch's postulates were completed on susceptible host plants (modified due to inability to grow Peronospora tabacina in pure culture). This was performed by suspending sporangia from cocultures in sterile water, as previously described, then, with a hypodermic syringe equipped with a 25 -gauge needle, infiltrating them into leaf panels of cv. KY14 tobacco plants. The infiltration technique was employed due to quarantine conditions imposed in the lab during the tobacco growing season. The plants were observed daily for disease symptoms (chlorosis). Koch's postulates were deemed complete after sporulation was induced on the newly infected plants and was checked microscopically for sporangia and sporangiophore morphology of Peronospora tabacina.

Regeneration of plants from cocultures, testing for resistance, and assessing pathogen presence. Differentiated shoots from cocultures were excised aseptically with a sterile scalpel and placed (excision side down) into MS- medium for induction of roots. Plantlets forming roots after 2 to 3 weeks were taken from plates and potted as described previously for host material (except that a plastic bag was placed over the pots to minimize water loss through transpiration). Within 4 to 5 weeks, plants that successfully regenerated from infected cocultures were challenged by spray inoculation with a $5 \times 10^{3}$ spores per $\mathrm{ml}$ suspension of Peronospora tabacina sporangia, applied over the entire surface of each plant until runoff. Susceptible control plants grown from seed and of the same age were inoculated similarly. Plants regenerated from uninfected tissue culture, in addition to plants grown from seed, were included as controls. Plants were then monitored over the course of 2 weeks for symptom development. Disease assessment was based on symptom comparison with inoculated controls. Plants successfully regenerated from the cocultures were tested for pathogen presence, prior to testing for resistance, by introducing them into conditions favorable for sporulation $\left(17\right.$ to $23^{\circ} \mathrm{C}$, $>95 \%$ humidity, and overnight darkness), followed by observations for signs of the pathogen (sporulation). To detect possible asymptomatic infections, a polymerase chain reaction (PCR)-based procedure (17) was employed on tissue from at least 20 plants selected at random from both host species. As a negative control, PCR diagnosis was carried out on tissue from at least five plants regenerated from uninfected tissue cultures. Positive controls consisted of PCR diagnosis on Peronospora tabacina-infected leaf tissue from both $N$. tabacum and $N$. repanda.

\section{RESULTS}

The primary objective of this study was to maintain infections of Peronospora tabacina for extended periods in callus from wild and commercial tobacco species. Cocultures were initially generated from surface-sterilized $N$. tabacum leaf tissue 5 days after inoculation with Peronospora tabacina. There was no indication of contamination in 20 out of 25 cocultures. The 20 uncontaminated cultures were investigated further. After 1 month, the pathogen was induced to sporulate by overnight darkness (Fig. 1) and sporangia were collected from the cocultures and suspended in water. Because of relatively low amounts of sporulation on cocultures derived from surface-sterilized infected leaf tissue (typically less than $10 \%$ of the surface area), the spore suspensions contained an estimated 500 spores per $\mathrm{ml}$. These suspensions were used to generate new infections (gnotobiotic associations) on axenic $N$. tabacum and $N$. repanda derived from surface-sterilized seed. New infections were evident on leaves of inoculated axenic plants of both host species within 10 days and were characterized by chlorotic lesions. The new infections on axenic host plants were
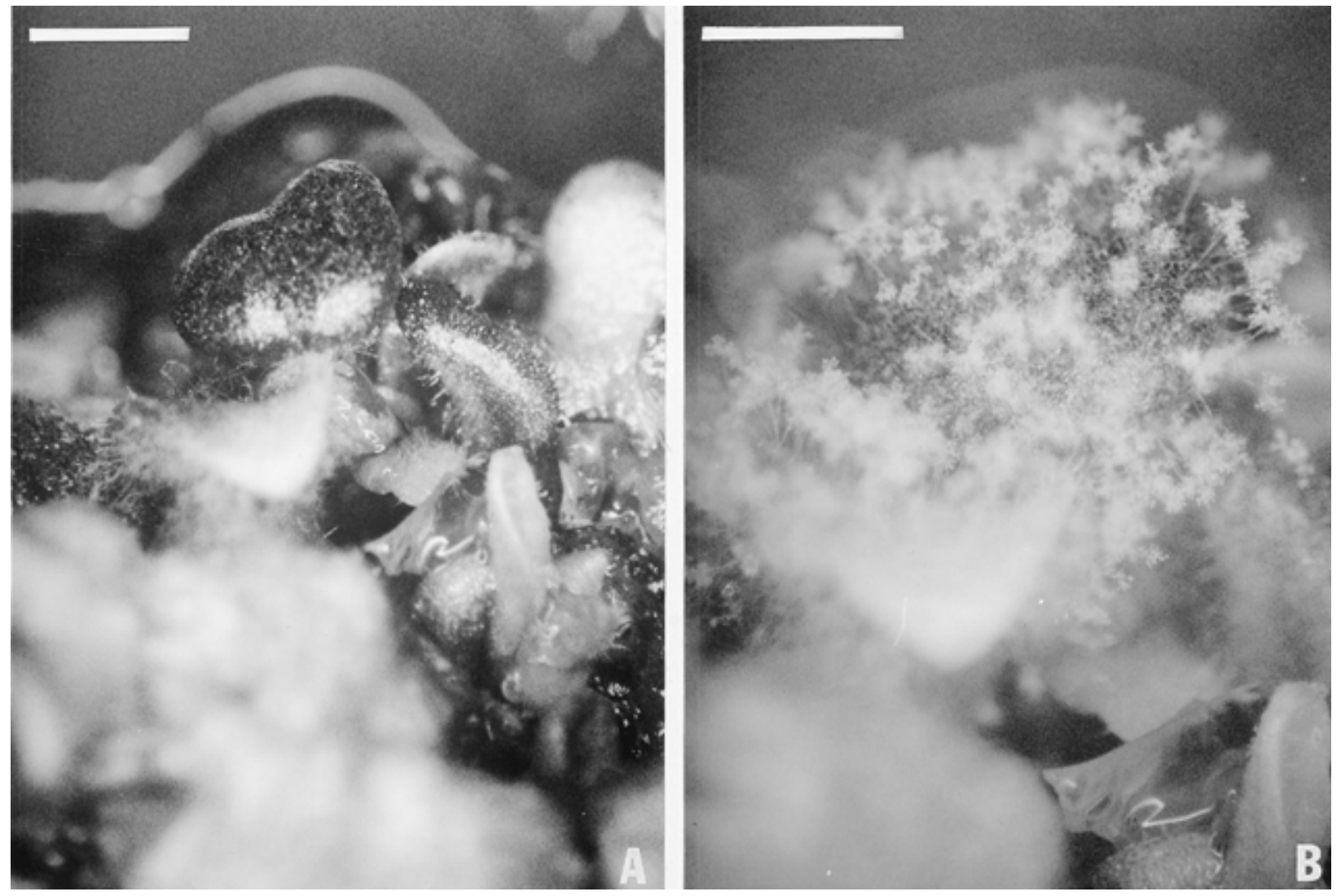

Fig. 1. A, Coculture prior to induction of sporulation and $\mathbf{B}$, following induction of sporulation, displaying multiple sporangiophores bearing asexual sporangia. Bars $=1 \mathrm{~mm}$. 
used as a source of infected tissue to generate new cocultures without the need for surface sterilization. These new cocultures were grown in two light regimes including $23 \mathrm{~h}$ light and $24 \mathrm{~h}$ dark. After the first $24 \mathrm{~h}$ in coculture, sporulation occurred on all tissue grown in $24 \mathrm{~h}$ dark, as expected, and hyphae emerged from the tissue grown in $23 \mathrm{~h}$ light. Furthermore, sporangia from the gnotobiotic associations were used to inoculate uninfected callus, successfully generating new cocultures.

We found that the pathogen could be maintained in coculture with either $N$. tabacum or $N$. repanda callus for periods of up to 1 year if they were maintained in the dark with biweekly subculture. To check for pathogen viability, sporulation was induced on several of the light-grown cocultures throughout the course of this study by placing in overnight darkness. Light-grown cocultures with $N$. tabacum or $N$. repanda callus harbored the pathogen for up to 4 or 8 months, respectively, providing sporulation was not previously induced. Sporulation on light-grown cocultures typically covered 50 to $70 \%$ of the surface. In some specimens, sporulation covered the entire surface following induction. The resulting sporangia were collected and used to complete Koch's postulates, indicating survival of Peronospora tabacina.

Induction of sporulation on light-grown cocultures with $N$. tabacum callus resulted in necrosis within 2 weeks after sporulation was induced. On cocultures with $N$. repanda callus, necrosis occurred within 1 month after induced sporulation. Necrosis varied depending on the amount of sporulation and was not observed on parts of the cocultures that lacked signs of the pathogen. Necrosis was not observed on light-grown cocultures not induced to sporulate.

In cocultures grown in darkness and subcultured biweekly, the pathogen continuously sporulated throughout the course of 1 year, but sporulation was scant (only a few scattered sporangiophores) in comparison to induced sporulation on light-grown cocultures. Furthermore, little or no necrosis was observed in the dark-grown cocultures.

Sporulation of the pathogen was observed on both nondifferentiated callus (Fig. 2A) and on differentiated shoots (Fig. 2B) in cocultures of both host species. This occurred on both light-grown cocultures that were induced to sporulate and on dark-grown cocultures in which sporulation occurred continuously. In each case, Koch's postulates were completed with the sporangia.

Sporangia of Peronospora tabacina normally appear lemon or balloon shaped (Fig. 3A), but sporangia and sporangiophores of abnormal appearance were observed on several of the light-grown cocultures that were not placed under conditions conducive for sporulation. These sporangia differed from normal morphology in being "peanut" or "foot" shaped (Fig. 3B). Abnormal sporangiophores were much longer ( $>1 \mathrm{~mm}$ ) than the 400 to $750 \mu \mathrm{m}$ associated with typical sporangiophores of Peronospora tabacina. Production of abnormal sporangiophores and sporangia was scant (only a few widely scattered structures). In addition, the abnormal sporangia did not readily detach from the sporangiophores, determined by their absence on the medium surrounding the cocultures. Conversely, the medium surrounding cocultures that were induced to sporulate was typically covered with sporangia. Necrosis was not observed following abnormal sporulation. Normal sporangia and sporangiophore production could be induced by placing these cocultures in the dark overnight.

Interestingly, Peronospora tabacina hyphae grew from the host tissue onto the medium from dark-grown cocultures with $N$. repanda (Fig. 4A), but not in N. tabacum cocultures or light-grown $N$. repanda cocultures. This was most evident in cocultures that were maintained without biweekly subculturing. In some cases, sporangiophores with asexual sporangia could be seen emerging from within the mycelium that was growing on the medium (Fig. 4B). Sporangia were collected from these sporulation sites and were used to complete Koch's postulates. Mycelia were observed to grow up to $2 \mathrm{~cm}$ within the first 4 months from cocultures with
$N$. repanda as host component that were not subcultured biweekly, after which time no further growth was observed. These coenocytic (aseptate) hyphae resembled those of Phytophthora or Pythium spp. To test the possibility of contamination by Phytophthora or Pythium spp., which are both capable of saprophytic growth and can be systemic pathogens of tobacco, agar plugs with mycelium were transferred to various nutrient media, including MS+, MS-, PDA, NA, and V8-juice agar, and resulted in no further growth of the mycelium. In contrast, an isolate of Phytophthora parasitica pv. nicotianae from tobacco with black shank and an available Pythium isolate from Chrysanthemum grew actively on the aforementioned media within 1 week following inoculation.

After 45 days in coculture with biweekly subculture, shoots formed from the calli of both host species. Several of the shoots, both with and without signs (hyphae) of the pathogen, were excised and placed in MS- medium to induce root formation. In total, $476 \mathrm{~N}$. tabacum and $47 \mathrm{~N}$. repanda plants were regenerated from cocultures and none were shown to harbor the pathogen as determined by attempts at inducing sporulation. None of the shoots displaying signs of the pathogen at the time of excision from cocultures were successfully regenerated due to apparent overgrowth of pathogen and necrosis. To check for asymptomatic Peronospora tabacina infection of regenerants, PCR diagnosis was employed and resulted in all negatives, except for PCR on infected tissue (positive controls). Likewise, PCR results were negative on plants regenerated from control tissue cultures. To test

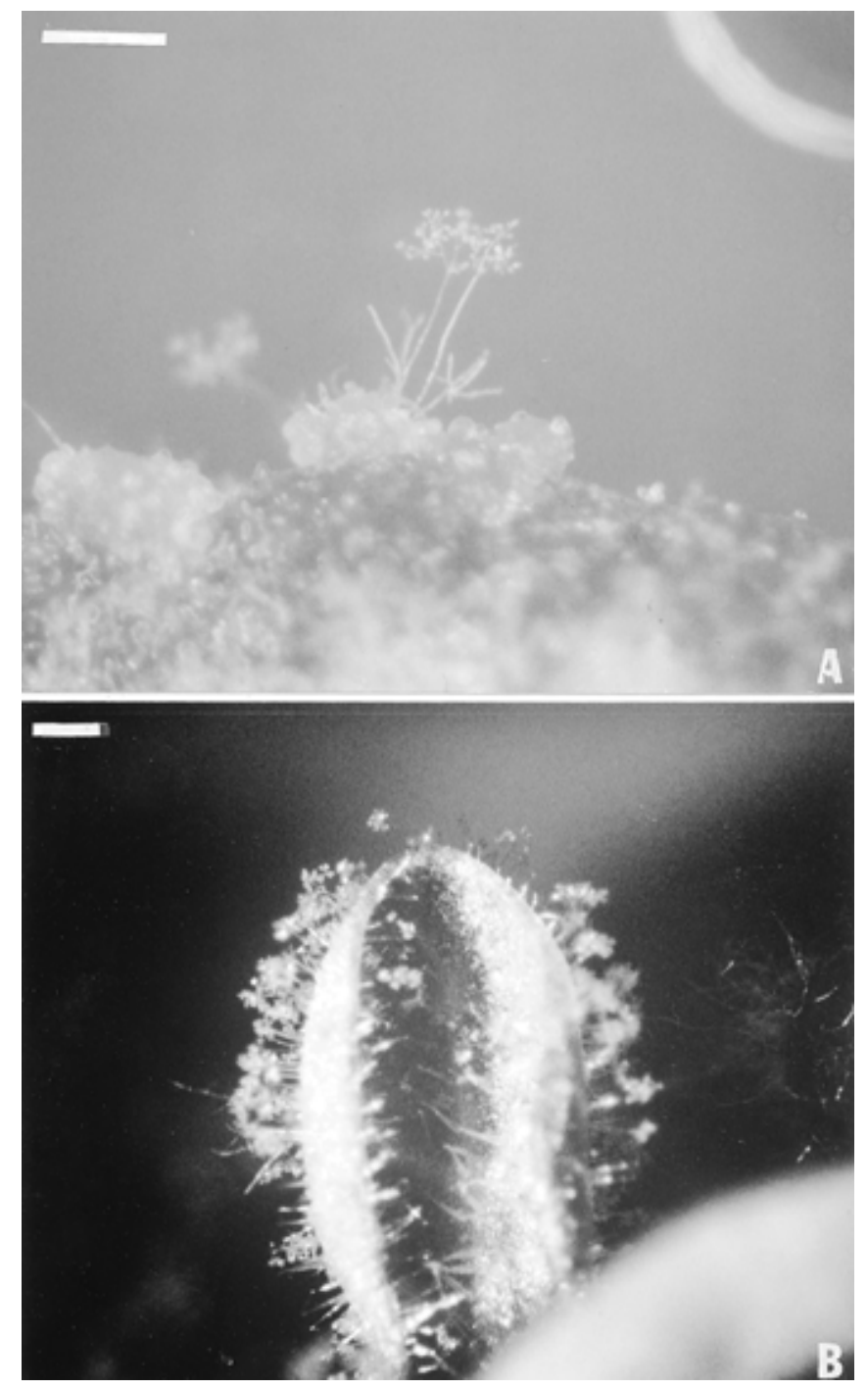

Fig. 2. Sporulation of Peronospora tabacina on A, nondifferentiated callus tissue and on $\mathbf{B}$, differentiated shoots in cocultures. Bars $=0.5 \mathrm{~mm}$. 
for any resistance that may have resulted from somaclonal variation or by selection imposed by the pathogen, we challenge-inoculated all regenerants alongside susceptible plants grown from seed or generated from uninfected tissue cultures. Only two plants regenerated from cocultures with $N$. tabacum as the host component showed resistance, which was characterized by necrotic flecking (HR-like, due to its superficial resemblance of a hypersensitive response) in response to infection and resulted in no observable
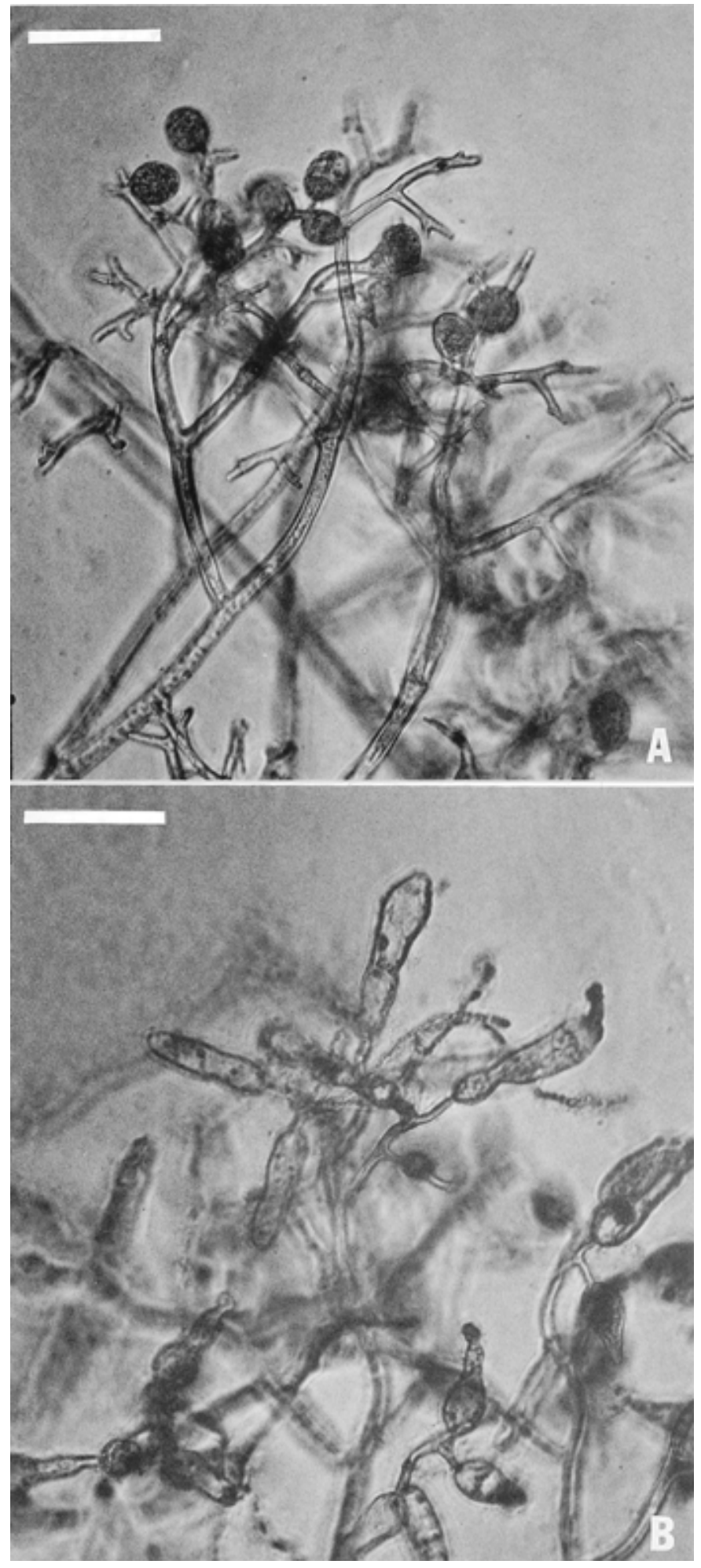

Fig. 3. A, Normal sporangia of Peronospora tabacina produced on cocultures following induction of sporulation in overnight darkness, and $\mathbf{B}$, unusual sporangia produced on cocultures grown in continuous 23 -h light. Bars $=50 \mu \mathrm{m}$. sporulation following induction. These two plants were grown to seed and more than 100 progeny from each were tested for HRlike response following challenge inoculation with Peronospora tabacina spores. No progeny exhibited any resistance to Peronospora tabacina.

None of the cocultures derived from gnotobiotic associations became contaminated as determined by visual and microscopic inspection and absence of fungal or bacterial growth in test media. The same was true for sporangia that were tested from the cocultures. However, there were several instances in which $N$. tabacum plants derived from surface-sterilized seed, whether inoculated with Peronospora tabacina or not, were obviously contaminated with either a pink-pigmented bacterium or a yellow-pigmented bacterium and were, therefore, discarded. The pink-pigmented bacterium was a gram-negative rod, whereas the yellow-pigmented bacterium was a gram-positive rod. No such bacteria were observed in any of the inoculated or uninoculated $N$. repanda plants derived from surface-sterilized seed. Both types of bacteria actively grew in NB, PDB, PDA, and NA. Negative controls consisting of uninfected axenic leaf tissue from both $N$. tabacum and $N$. repanda plants inoculated into the aforementioned media were negative for contamination. Positive controls consisting of Peronospora tabacina-infected tobacco leaf tissue from the greenhouse resulted in outgrowth of several different bacteria and fungi in all inoculated media.

\section{DISCUSSION}

This study was initiated to obtain axenic Nicotiana-Peronospora tabacina interactions to allow studies of the pathosystem, including infection, sporulation, and host responses, without confounding influences of other microorganisms. Methods for generating and maintaining dual Nicotiana-Peronospora tabacina associations were established and refined throughout this study. We began generating cocultures from surface-sterilized infected leaf material and found that this material could be used to initiate cocultures without subsequent contamination. Infected host tissue grown in nonaxenic growth chamber conditions was prone to colonization by opportunistic microorganisms, especially fungi, when infections were allowed to progress for more than 5 days, so it was necessary to use newly developed lesions as source material. Once contaminant-free inoculum was obtained from the cocultures, we were able to use sporangia from those associations to inoculate axenic host plants, subsequently generating gnotobiotic associations between Peronospora tabacina and $N$. tabacum or $N$. repanda. This was helpful because, in the absence of contaminating microorganisms, we could bypass the surface sterilization step in subsequent experiments to generate cocultures.

Although we were able to maintain the pathogen in coculture with host material for over a year, biweekly transfer of infected material to fresh MS- medium was performed. It is possible that the cocultures could be maintained successfully if subcultured less frequently. However, we did not explore this possibility. From the standpoint of maintaining clean inoculum, maintenance of the pathogen in gnotobiotic associations with axenic host plants seems as effective and more convenient than maintaining cocultures. However, one must first obtain contaminant-free inoculum, which we have shown, can be obtained from the cocultures.

One possible explanation for the ability of Peronospora tabacina to survive in dark-grown cocultures for the duration of this study is that the sporangia produced may cause repeated infections. In the light-grown cocultures, normal sporulation of the pathogen was suppressed by $23 \mathrm{~h}$ light, whereas sporulation occurred continuously in the dark-grown cocultures. We speculate that the continuous sporulation and subsequent germination of newly formed sporangia could have resulted in new infections on uninfected portions of the cocultures that might otherwise have remained pathogen free. 
Earlier studies involving dual Peronospora tabacina-N. tabacum tissue cultures employed methods in which the associations were derived by inoculating callus tissue from various Nicotiana spp. with sporangia from Peronospora tabacina $(5,14)$. These studies reported only sparse colonization by the fungus and little, if any, sporulation. Also, neither study resulted in long-term survival of both host and pathogen. We attempted such culturing in preliminary tests and found bacterial and fungal problems (rot), almost certainly due to contamination of the inoculum. Other possible reasons for previous failures to maintain long-term cocultures are (i) genotypic differences in host material used, (ii) differences in isolates of Peronospora tabacina used, and (iii) potentially low inoculum density resulting from the inoculation procedure (1).

Symptom development (chlorosis and necrosis) appeared to be slower in gnotobiotic associations compared with infected plants that were nonaxenic (grown in a soil mix). This might suggest that pathogenic or opportunistic microorganisms speed up the onset of symptom development associated with Peronospora tabacina infections in nonaxenic environments.

The onset of necrosis was much slower on induced light-grown $N$. repanda cocultures than on similar light-grown $N$. tabacum cocultures. These results are indicative of a much higher level of tolerance in $N$. repanda to infection by Peronospora tabacina than $N$. tabacum, as reported in previous whole plant studies (11).

Little or no observed necrosis in dark-grown cocultures of either host species might suggest that photosynthetically derived factors are required for necrosis to occur. Another possibility is that necrosis is associated with the amount of pathogen growth or sporulation that occurred on the cocultures. For example, in induced light-grown cocultures, the pathogen produced many more sporangiophores that covered much more of the callus than did the sparse sporulation on dark-grown cocultures. This question can be addressed in the future by comparative microscopic examinations.

Another interesting observation was the ability of Peronospora tabacina to grow on the medium from dark-grown cocultures with $N$. repanda as the host component. Although there have been similar reports for coculture of other downy mildew pathogens (13), to our knowledge, this is the first observation that Peronospora tabacina can produce sporangiophores from a nonplant surface. The absence of pathogen growth on the medium around light-grown cocultures of $N$. repanda might suggest that factors relating to light or photosynthesis affect pathogen growth. Because commercial tobacco transplants are often shipped in boxes and kept in the dark for days, this could have epidemiological significance as well.

We were also interested in the possibility of using cocultures to select for variant plants that may have developed heritable resistance to blue mold. Out of 476 regenerants from $N$. tabacum cocultures, only two plants showed some resistance and none of their seed progeny were resistant to blue mold. This result may corroborate earlier work in which tissue from tobacco plants inoculated with Peronospora tabacina (providing systemic protection) was grown in tissue culture for subsequent plant regeneration and resistance testing (8). It was shown that in plants regenerated from protected tissue there was no transmissibility of protection observed in plants from which the tissue was collected. Tuzun and Kuc (15) demonstrated that progeny from plants that had similar protection were not resistant when compared with inoculated controls.

Plants successfully regenerated from cocultures of both host species did not harbor the pathogen. Plantlets displaying signs of the pathogen at the time of excision from cocultures did not form roots. Instead, they were overgrown by Peronospora tabacina hyphae, which was followed by necrosis. One explanation is that excision from the cocultures together with infection exerts high levels of stress on the tissue, which, in turn, leads to necrosis. One question that arises might then be: Why do infected plantlets be- come overgrown by the pathogen and necrotic, whereas similarly infected cocultures remain comparatively necrosis-free and in most cases are not overgrown by the pathogen? One possibility might relate to differences between the two types of media used to grow cocultures and to induce roots on excised shoots. Cocultures were grown on a medium that contains growth hormones (IAA and $\mathrm{BAP}$ ), and the medium used to induce roots has none. It is possible that the tissue must be actively growing, as is the case with cocultures in the presence of growth hormones, or they will be overgrown by the pathogen, hastening necrosis. Thus, the time required for root formation may have exceeded the time required for the pathogen to thoroughly ramify the host tissue.

Although cocultures were generally free of culturable contaminants, there were two different bacteria found in association with some $N$. tabacum seeds. These were (i) a pink-pigmented, gramnegative, rod-shaped bacterium, and (ii) a yellow-pigmented, gram-positive, rod-shaped bacterium that grew from the bases of some $N$. tabacum plants derived from surface-sterilized seed. Because we selected only plants with no observable contamination for use in our study, we avoided introduction of these bacteria into our coculture and gnotobiotic systems. It is possible that the pink bacterium was a pink-pigmented facultative methylotroph (PPFM), reported to be ubiquitous in many plant species, where they establish epiphytically and are sometimes seed transmitted $(2,16)$. We also noted that these bacteria grew in the presence of the antibiotics carbenicillin and cefotaxime. Cefotaxime has been previ-

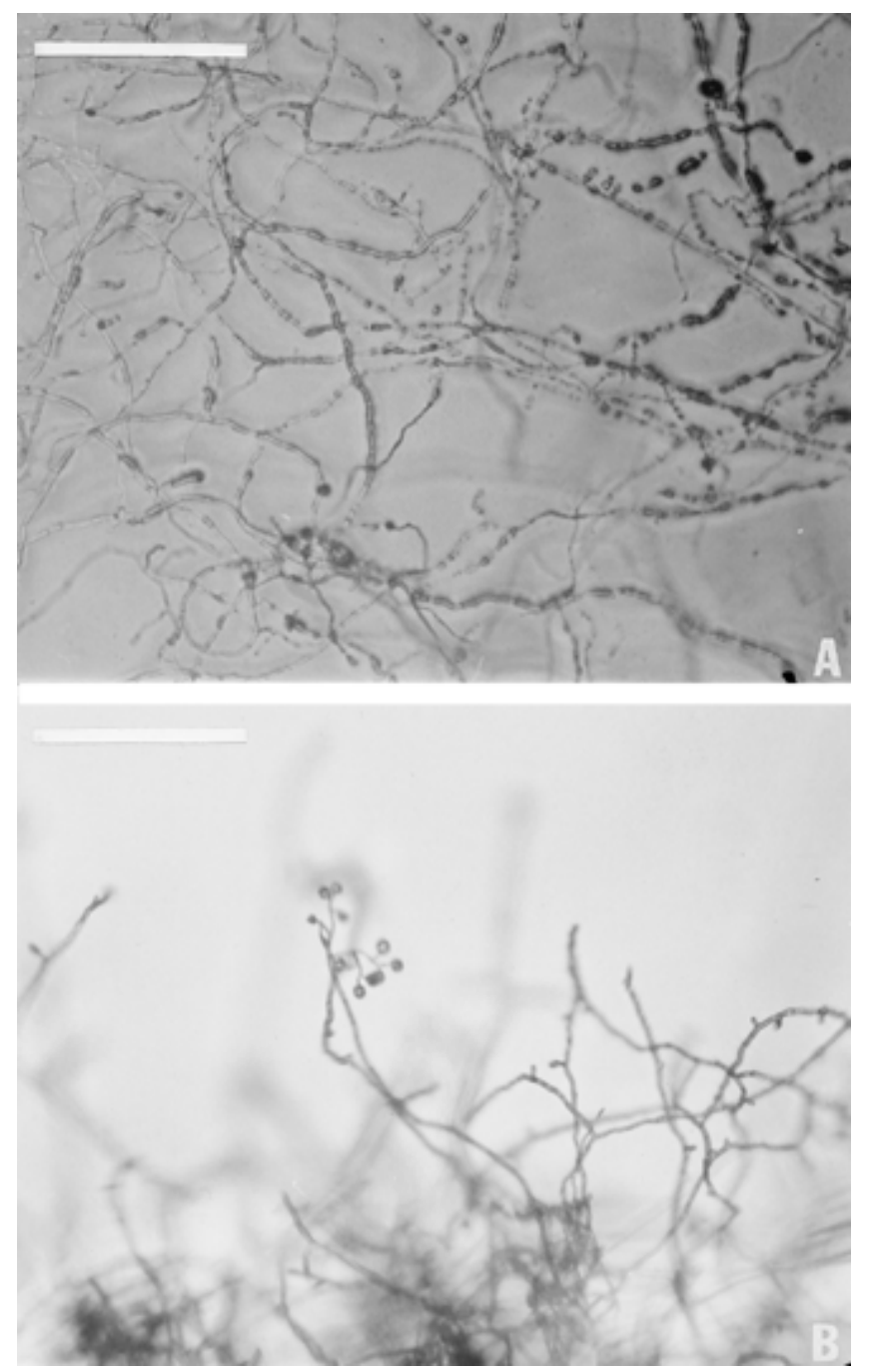

Fig. 4. A, Mycelium of Peronospora tabacina growing on the medium from dark-grown cocultures, with Nicotiana repanda as a host component, and $\mathbf{B}$, sporulating within mycelium. Bars $=0.5 \mathrm{~mm}$. 
ously reported to inhibit PPFMs (2). However, it is possible that the antibiotics could have degraded over the time necessary for seed germination and seedling development.

In summary, long-term cocultures of Peronospora tabacina and both wild and cultivated Nicotiana spp. have been established. We demonstrated that Peronospora tabacina can successfully colonize and sporulate on both undifferentiated callus and differentiated shoots in cocultures. Conditions that induced Peronospora tabacina sporulation in the cocultures were similar to those that induce sporulation in plants grown from seed, with respect to temperature, humidity, and darkness, indicating some physiological similarity of the coculture and whole plant systems. Also, contaminant-free inoculum generated via coculture will be useful for future studies on biochemical aspects of the pathogen, an area of research that has been, to this point, confounded by the presence of contaminating microbes. Thus, the techniques described herein should establish a new standard for research with Peronospora tabacina, because most, if not all, previous work has been carried out under nonaxenic conditions.

\section{ACKNOWLEDGMENTS}

This research was supported in part by the Tobacco and Health Research Institute at the University of Kentucky, and R. J. Reynolds Company. E. P. Heist was the recipient of a Jeffrey Fellowship from the University of Kentucky. This is publication 00-12-170 of the Kentucky Agricultural Experiment Station, published with the approval of the director. We thank S. Dutton for expert technical assistance in handling and maintaining Peronospora tabacina isolates and G. Collins and his coworkers, C. Redmond and C. Meurer, for assistance and suggestions regarding tissue culture materials and procedures.

\section{LITERATURE CITED}

1. Amerson, H. V., and Mott, R. L. 1978. Technique for axenic production and application of Cronartium fusiforme basidiospores. Phytopathology 68:673-675.

2. Chanprame, S., Todd, J. J., and Widholm, J. M. 1996. Prevention of pink-pigmented methylotrophic bacteria (Methylobacterium mesophilicum) contamination of plant tissue cultures. Plant Cell Rep. 16:222-225.

3. Clayton, E. 1945. Resistance of tobacco to blue mold (Peronospora tabacina). J. Agric. Res. 70:79-87.

4. Ingram, D. S., and Helgeson, J. P. 1980. The establishment of dual cultures of downy mildew fungi and their hosts. Pages 139-145 in: Tissue Culture Methods for Plant Pathologists. Blackwell Scientific Publications, Oxford.

5. Izard, C., Lacharpagne, J., and Schiltz, P. 1964. Comportement de Peronospora tabacina dans les cultures de tissus et role de l'epiderme foliare. SEITA (Serv. Exploit. Ind. Tab. Alumettes) Ann. Dir. Etud. Equip. Sect. 2:95-99.

6. Lucas, G. B. 1975. Black shank. Pages 115-141 in: Diseases of Tobacco. Biological Consulting Associates, Raleigh, NC.

7. Lucas, G. B. 1975. Damping-off. Pages 161-172 in: Diseases of Tobacco. Biological Consulting Associates, Raleigh, NC.

8. Lucas, J. A., Dolan, T. E., and Coffey, M. D. 1985. Nontransmissibility to regenerants from protected tobacco explants of induced resistance to Peronospora hyoscyami. Phytopathology 75:1222-1225.

9. Morel, G. 1944. Le development du mildiou sur des tissus de vigne cultives in vitro. C. R. Acad. Sci. 218:50-52.

10. Murashige, T., and Skoog, F. 1962. A revised medium for rapid growth and bioassays with tobacco tissue culture. Physiol. Plant 15:473-497.

11. Reuveni, M., Nesmith, W. C., and Siegel, M. R. 1986. Symptom development and disease severity in Nicotiana tabacum and $N$. repanda caused by Peronospora tabacina. Plant Dis. 70:727-729.

12. Reuveni, M., Nesmith, W. C., and Siegel, M. R. 1988. Virulence of an endemic isolate of Peronospora tabacina from Texas to Nicotiana tabacum and $N$. repanda. Plant Dis. 72:1024-1027.

13. Tiwari, M. M., and Arya, H. C. 1969. Sclerospora graminicola axenic culture. Science 163:291-293.

14. Trigiano, R. N., Van Dyke, C. G., Spurr, H. W., Jr., and Grey, D. J. 1984. Infection and colonization of tobacco callus by Peronospora tabacina. Phytopathology 74:280-285.

15. Tuzun, S., and Kuc, J. 1987. Persistence of induced systemic resistance to blue mold in tobacco plants derived via tissue culture. Phytopathology 77:1032-1035.

16. Widholm, J. M. 1996. The rare occurrence of plant tissue culture contamination by Methylobacterium mesophilicum. Plant Cell Tissue Organ Cult. 45:201-205.

17. Wiglesworth, M. D., Nesmith, W. C., Schardl, C. L., Li, D., and Siegel, M. R. 1994. Use of specific repetitive sequences in Peronospora tabacina for the early detection of the tobacco blue mold pathogen. Phytopathology 84:425-430.

18. Wolf, F. A. 1947. Tobacco downy mildew, endemic to Texas and Mexico. Phytopathology 37:721-729.

19. Wolf, F. A., Dixon, L. F., McLean, R., and Darkis, F. R. 1934. Downy mildew of tobacco. Phytopathology 24:337-363. 